\title{
Clinical and epidemiological characterization of psoriasis and psoriatic arthritis in a multidisciplinary assessment model
}

Background: Presentation and clinical course in Psoriasis are heterogeneous. A multidisciplinary assessment model was designed, under a procedure manual for five specialties related to disease manifestations: dermatology, rheumatology, internal medicine, nutrition and psychiatry, to evaluate at the same time and place, patients with psoriasis, to determine the frequency of psoriatic arthritis and other comorbidities in patients with psoriasis, as well as performing a clinical and epidemiological characterization of psoriasis and psoriatic arthritis in patients seeking medical consultation in a multidisciplinary assessment model in Guadalajara Jalisco, Mexico.

Methods and Findings: A prospective study that included patients that attended a multidisciplinary psoriasis clinic from August 2016 to February 2018. Patient triage and evaluation of psoriasis or psoriatic arthritis was performed either by a rheumatologist or a dermatologist at an appointment prior to enrollment. If a clinical, radiological or histological criterion was met, patient was scheduled to an appointment to the multidisciplinary clinic. Fifty three patients were included. Median age was 42 years old and $62.3 \%$ were female. Family history of psoriasis was found in $26.4 \%$ of population and $19.4 \%$ had spondyloarthropathy family history. Most common comorbidities found were overweight and obesity $66 \%$, metabolic syndrome in $35.8 \%$, diabetes mellitus in $15.1 \%$, dyslipidemia in $22.6 \%$, hypertension in $13.1 \%$ and fatty liver in $3.8 \%$. Affective assessment was performed and $49.1 \%$ met criteria for anxiety Psoriasis was found in $96.2 \%$ and $56.6 \%$ in nails. PASI was moderate to severe in $49.1 \%$. Psoriatic arthritis in $58.5 \%$ of patients that met CASPAR criteria. Axial disease was present in $51.6 \%$, peripheral joint disease in $71 \%$ and in $13.2 \%$ both. Enthesitis was present in $54.8 \%$ and $16.1 \%$ had dactylitis. DAS28 was performed and showed $45.2 \%$ with severe activity. Total number of subjects with peripheral disease did not met criteria for remission measured by DAS28 or MDA.

Conclusions: We found that nearly half of the patients have moderate to severe form of psoriasis. The number of patients with psoriatic arthritis was higher than expected with both axial and peripheral manifestations. We have found a high proportion of patients with comorbidities such as metabolic syndrome, diabetes mellitus, hypertension, dyslipidemia and obesity. We observed a high frequency of psychiatric illness like depression and anxiety. The limitations of our study are the small sample to highlight if comorbidities had an impact on the severity of skin and joint disease. This multidisciplinary model has identified psoriasis as a disease with an unpredictable course, which requires several evaluations by a multidisciplinary model, with a group of experts beyond Dermatologist and Rheumatologist.

Keywords: centro de investigación en enfermedades autoinmunes • psoriatic arthritis • erythrocyte sedimentation rate $\cdot$ reactive protein $c \cdot$ psoriasis $\bullet$ dermatology quality of life index $\bullet$ psoriasis area and severity index $\cdot$ body surface area $\cdot$ nail psoriasis severity index

Abbreviations: CINEA: Centro de Investigación en Enfermedades Autoinmunes; PsA: Psoriatic Arthritis; ESR: Erythrocyte Sedimentation Rate; PCR: Reactive Protein C; Pso: Psoriasis; DLQI: Dermatology Quality of Life Index; PASI: Psoriasis Area and Severity Index; BSA: Body Surface Area; NAPSI: Nail Psoriasis Severity Index; CASPAR: Classification Criteria for Psoriatic Arthritis; BASDAI: Bath Ankylosing Spondylitis Disease Activity Index; BASMI: Bath AS Metrology Index; BASFI: Bath AS Functional Index; DAS 28: Disease Activity Score 28; LEl: Leeds Enthesitis Index; MDA: Minimal Disease Activity

\section{Alejandra Lopez $\mathrm{R}^{* 1}$, Karla Macias $\mathbf{G}^{2}$, Marcela Cerda $\mathrm{E}^{3}$ \& Pamela Sandoval $\mathbf{M}^{3}$ \\ ${ }^{1}$ Council of Rheumatology Certified, Multidisciplinary Model Psoriasis and Psoriatic Arthritis Clinic Co-director, Principal Investigator in Clinical Trials in Rheumatology CINEA, Guadalajara Jalisco, México \\ ${ }^{2}$ Mexican Council of Dermatology Certified, Centro Dermatológico del Country Director, Multidisciplinary Model Psoriasis and Psoriatic Arthritis Clinic Director, Principal Investigator in Clinical Trials in CINEA, Guadalajara Jalisco, México ${ }^{3}$ Multidisciplinary Model Psoriasis and Psoriatic Arthritis Clinic Team, Mexican Council of Dermatology Certified, Mexico \\ *Author for correspondence: dra_alejandralopezrdz@hotmail.com}




\section{Introduction}

Psoriasis is a chronic, inflammatory systemic disease typically characterized by erythematous, scaly patches, or plaques on the skin resulting from hyperploriferation of epidermal keratinocytes [1]. The disorder affects approximately 1-3\% of the world population [2]. Psoriatic arthritis (PsA) is a spondyloarthritic condition, which is present in up to $42 \%$ of individuals with psoriasis and up to $15 \%$ of patients with psoriasis may have undiagnosed PsA [3]. Additionally, the incidence of PsA in patients with psoriasis is 1.87 per 100 [4]. The clinical manifestations of PsA are complex and heterogeneous. Symptoms of PsA include joint pain and stiffness, skin and nail psoriasis, dactylitis, and persistent, painful enthesitis [5]. Although PsA was once considered a mild disease, it is now understood that progressive structural damage can begin early in the course of PsA [5]. Damage is evidenced by radiologic changes in up to $47 \%$ of patients at a median interval of 2 years and causes irreversible disability [6]. Emerging data also suggest associations between psoriasis and psoriatic arthritis, including hypertension, dyslipidemia, or diabetes and other chronic diseases like kidney disease, inflammatory bowel disease, hepatic disease [7]. Recognizing the comorbid disease burden of psoriasis is essential for ensuring comprehensive care of patients with psoriasis. The clinical implications of the comorbid diseases that are associated with psoriasis and psoriatic arthritis justify a multidisciplinary management. In order to avoid disease progression and associated long term complications, it is important to be aware of clinical and epidemiological characteristics that are associated with the severity of these medical conditions in Mexican population, and to our knowledge, epidemiological data are still not outlined. In the interest of addressing this problem, our study group designed and conducted a multidisciplinary assessment model that gathers 5 different medical specialties; rheumatology, dermatology, internal medicine, clinical nutrition and a psychiatrist to evaluate patients with psoriasis in the same place at the same visit.

\section{Methods}

\section{Study population}

Sample size was calculated using population proportion formula with the software OpenEPi version 3, assuming an expected frequency of $3 \%$, with $95 \%$ confidence interval. The sample size was 45 subjects, we recruit 53 patients. We identified patients with psoriasis or psoriatic arthritis in a routine medical visit of 3 dermatologists and 1 rheumatologist in a high specialty dermatology center in Guadalajara Jalisco Mexico, Dermatologico Country, using a previous algorithm based on the Procedures Manual (Figure 1). In a first visit, the dermatologists confirmed the diagnosis of psoriasis according to the clinical presentation or if the clinical diagnosis was doubtful, the patient underwent a skin biopsy to confirm the diagnosis of psoriasis. Regarding the evaluation of rheumatology, regular consultation patients were included if met CASPAR criteria for the diagnosis of psoriatic arthritis, and were always reviewed by the dermatologist for the skin diagnosis. If the patient complied with criteria of psoriasis or psoriatic arthritis, several studies were requested for the multidisciplinary evaluation in the psoriasis clinic that included: complete blood test, lipid profile, blood chemistry, rheumatoid factor, acute phase reactants as PCR or ESR, liver tests, X-rays of the pelvis, spine, hands and feet. During the appointment in the multidisciplinary clinic, a team was convened, consisting of 3 dermatologists, 1 rheumatologist, 1 internal medicine physician, 1 psychiatrist and 1 nutritionist. Each specialist, under strict steps of the procedures manual, was dedicated to assessing their area of interest in search of comorbidities associated with the underlying disease: internal medicine and nutrition evaluated metabolic syndrome, nutritional status, dyslipidemias, diabetes, hypertension and other associated diseases, as well as calculation of cardiovascular risk in all patients, in addition to issuing recommendations for the prevention or control according to the findings. Psychiatry applied validated scales of depression and anxiety as well as suicide ideation to identify psychiatric comorbidities in this type of patients with pharmacological interventions or psychotherapy as the case may be and finally dermatology and rheumatology in each of the patients applied the clinimetry according to specialty for better classification of patients as well as better understanding the manifestations of the disease and treatment decisions. Some of the validated scales used were DLQI, PASI, BSA, NAPSI, CASPAR, BASDAI, BASMI, BASFI, DAS 28, LEI, MDA. All subjects of the study signed an informed consent.

\section{Study design}

For qualitative variables such as psoriatic 


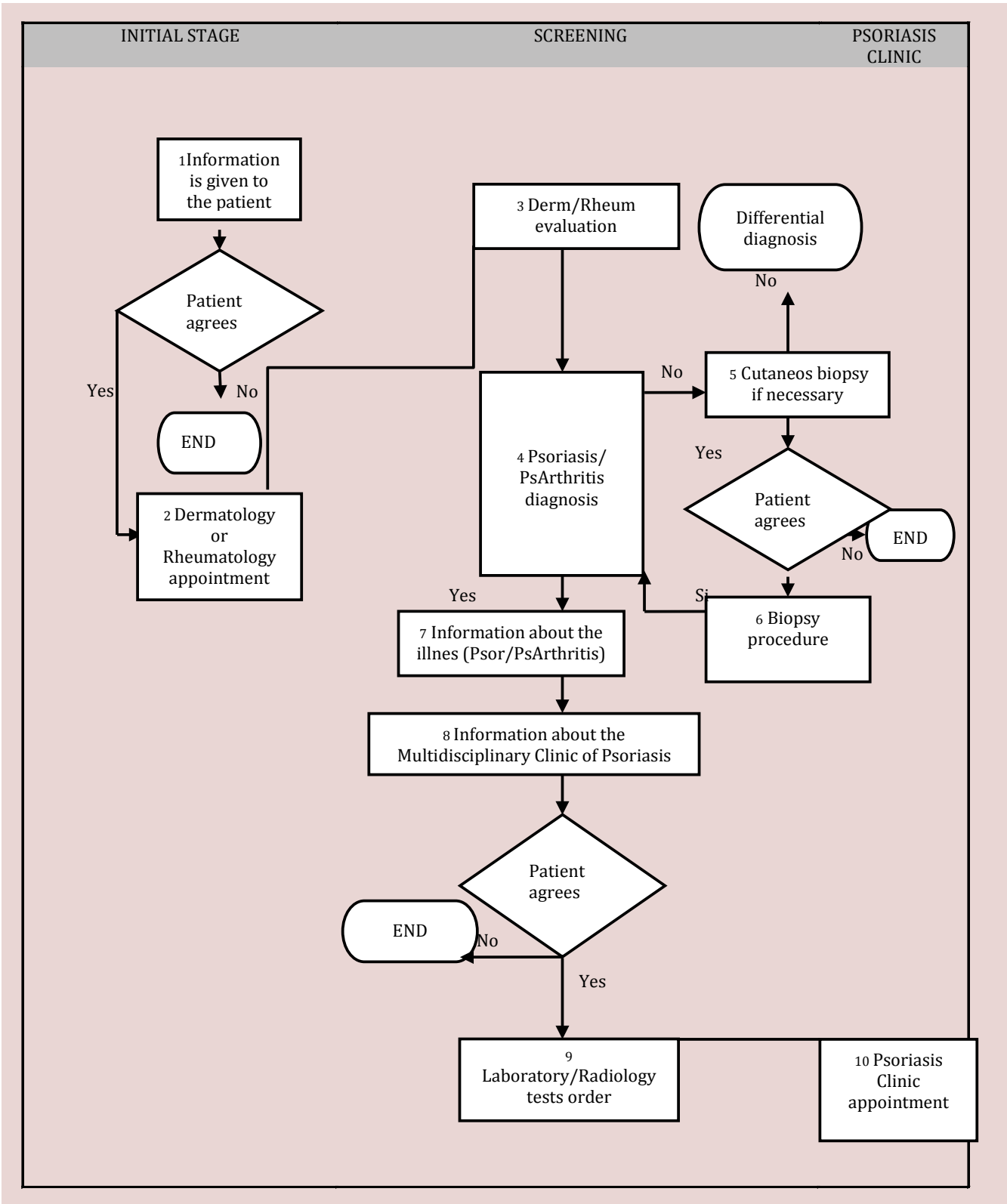

\section{Figure 1. First Visit Algorithm}

arthritis, sex, family history of psoriasis and spondyloarthropathies, smoking, alcoholism, exercise, healthy eating habits, nutritional status, comorbidities, presence of depression and anxiety, frequencies and percentages were calculated. For the quantitative variables such as age, score of PASI, BSA, NAPSI, DLQI, HAQ, LEI, BASDAI, BASMI, BASFI and DAS28, the median was calculated as a measure of dispersion, the interquartile range (RIQ 25.75). For the variables: total cholesterol, HDL cholesterol, having a normal distribution, the mean was calculated as a measure of central tendency and standard deviation as a measure of dispersion. To determine if there were differences between the patients diagnosed with psoriatic arthritis and without arthritis, the $\mathrm{X}^{2}$ test was used for qualitative variables, for the quantitative variables of normal distribution the Student's t test was used, for the quantitative variables of free distribution it was used the U Mann Whitney test. To determine the risk factors associated with the severity of psoriatic arthritis, we used a logistic regression model, which was adjusted for the variables metabolic syndrome, dyslipidemia, enthesitis and obesity, in all cases the OR and its 95\% CI were calculated. It was considered significative a $\mathrm{p}$ value $<0.05$. The software used was IBS SPSS Statistics 23. 


\section{Data collection}

All subjects were interviewed in a first medical visit performed by dermatologist or rheumatologist prior to attending the multidisciplinary psoriasis clinic in Dermatologico Country. In that visit medical record was carefully filled and detailed questions were made to obtain patient demographics, chief complaint, history of present illness, past medical history, family history, social history, allergies, medication history, review of systems and physical examination, all parts of a complete medical record, written in paper according to local legislation. If criteria were met for psoriasis or psoriatic arthritis, several laboratory and $\mathrm{X}$-ray tests were requested. With the results, the patient was evaluated in Multidisciplinary Clinic and filled questionnaires for severity of disease, like DLQI, BASDAI, BASFI, Depression and anxiety (HADS, EADG), Columbia (suicidal ideation) and were then evaluated with each specialty. After this visit, data base was filled in Microsoft Excel for Mac Version 15.21.1 in a MacBook Air with 189 variables, and for each one a numerical value was attributed.

\section{Results}

Table 1 shows the general characteristics of the population. A total of 53 participants were included, $62.3 \%$ of the participants corresponded to the female sex, $26.4 \%$ of the participants had a family history of psoriasis. The most frequent comorbidity in this population was metabolic syndrome that affected $35.8 \%$ of the participants. When assessing psychiatric comorbidity, we can observe that $30.2 \%$ of the participants met diagnostic criteria for depression and $49.1 \%$ of the population met diagnostic criteria for anxiety. Psoriasis was presented in $96.2 \%$ of the participants and $56.6 \%$ participants presented nail psoriasis. Table 2 shows the clinimetry of patients with psoriasis, $96.2 \%$ of the participants presented skin psoriasis and $56.6 \%$ presented nail psoriasis, $58.5 \%$ of the participants met diagnostic criteria for psoriatic arthritis. $50 \%$ of the population had psoriasis with a mild PASI and the other $50 \%$ a moderate-severe PASI. In Table 3 is possible to observe the general characteristics of patients with and without psoriatic arthritis. The antecedent of Spondyloarthropathy in relatives in the group with psoriatic arthritis was major $v s$. the group without psoriatic arthritis $(\mathrm{p}<0.05)$. Between both groups it was possible to observe differences in the ESR values, in the group of

\begin{tabular}{|c|c|}
\hline \multirow{2}{*}{\multicolumn{2}{|c|}{$n=53$}} \\
\hline & \\
\hline Age (years) a & $42(30,56.50)$ \\
\hline $\begin{array}{l}\text { Sex, } n(\%)^{b} \\
\text { Female }\end{array}$ & $33(62.3)$ \\
\hline Psoriasis FH, n (\%) ${ }^{\mathbf{b}}$ & $14(26.4)$ \\
\hline Spondyloarthropathy FH, n (\%) & $6(19.4)$ \\
\hline Tobacco use, n (\%) & $14(26.4)$ \\
\hline Physical inactivity, $\mathrm{n}(\%)^{\mathrm{b}}$ & $38(71.7)$ \\
\hline Poor eating habits, $\mathbf{n}(\%)^{b}$ & $41(77.4)$ \\
\hline \multicolumn{2}{|l|}{ Nourishment, $\mathrm{n}(\%)^{\mathrm{b}}$} \\
\hline Normal & $18(34)$ \\
\hline Overweight I & $6(11.3)$ \\
\hline Overweight II & $12(22.6)$ \\
\hline Obesity Class I & $11(20.8)$ \\
\hline Obesity Class II & $4(7.5)$ \\
\hline Obesity Class III & $2(3.8)$ \\
\hline \multicolumn{2}{|l|}{ Comorbidities $\mathbf{n}(\%)^{b}$} \\
\hline Diabetes Mellitus & $8(15.1)$ \\
\hline Hypertension & $7(13.2)$ \\
\hline Dyslipidemia & $12(22.6)$ \\
\hline Metabolic syndrome & $19(35.8)$ \\
\hline Fatty liver & $2(3.8)$ \\
\hline Goldberg scale for depression, $\mathrm{n}(\%)^{\mathrm{b}}$ & $16(30.2)$ \\
\hline Goldberg scale for anxiety, $\mathrm{n}(\%)^{\mathrm{b}}$ & $26(49.1)$ \\
\hline \multicolumn{2}{|l|}{ Psoriasis, $\mathbf{n}(\%)^{b}$} \\
\hline Skin psoriasis & $51(96.2)$ \\
\hline Nail psoriasis & $30(56.6)$ \\
\hline $\begin{array}{l}\text { Psoriatic arthritis, } n(\%)^{b} \\
\text { CASPAR criteria }\end{array}$ & $31(58.5)$ \\
\hline \multicolumn{2}{|c|}{$\begin{array}{l}\text { a Data showed as median and Inter Quartile Range ( } \\
25, P 75) \\
\text { b Data showed as frequency and percentage }\end{array}$} \\
\hline
\end{tabular}

patients with psoriatic arthritis a ESR $>15 \mathrm{~mm} /$ hr was observed in $74.2 \%$ of the participants compared to $27.3 \%$ of the participants without psoriatic arthritis $(\mathrm{p}=0.001)$. The $9.7 \%$ [3] of patients with psoriatic arthritis presented positive rheumatoid factor compared to $4.5 \%$ [1] in those without diagnosis of psoriatic arthritis $(p=0.633)$. No significant differences were observed for the variables of sex, family history of psoriasis, smoking, alcoholism, exercise, eating habits, nutritional status, comorbidities, depression, anxiety, glucose, total cholesterol, HDL cholesterol, LDL, triglycerides, leukocytes and rheumatoid factor. Table 4 shows the clinical characteristics of patients with psoriatic arthritis. According to the skin psoriasis activity it is possible to observe that the most frequent presentation corresponded to plaque psoriasis in $67.7 \%$ of the participants followed by guttate psoriasis in $9.7 \%$ of the participants, $67.7 \%$ 


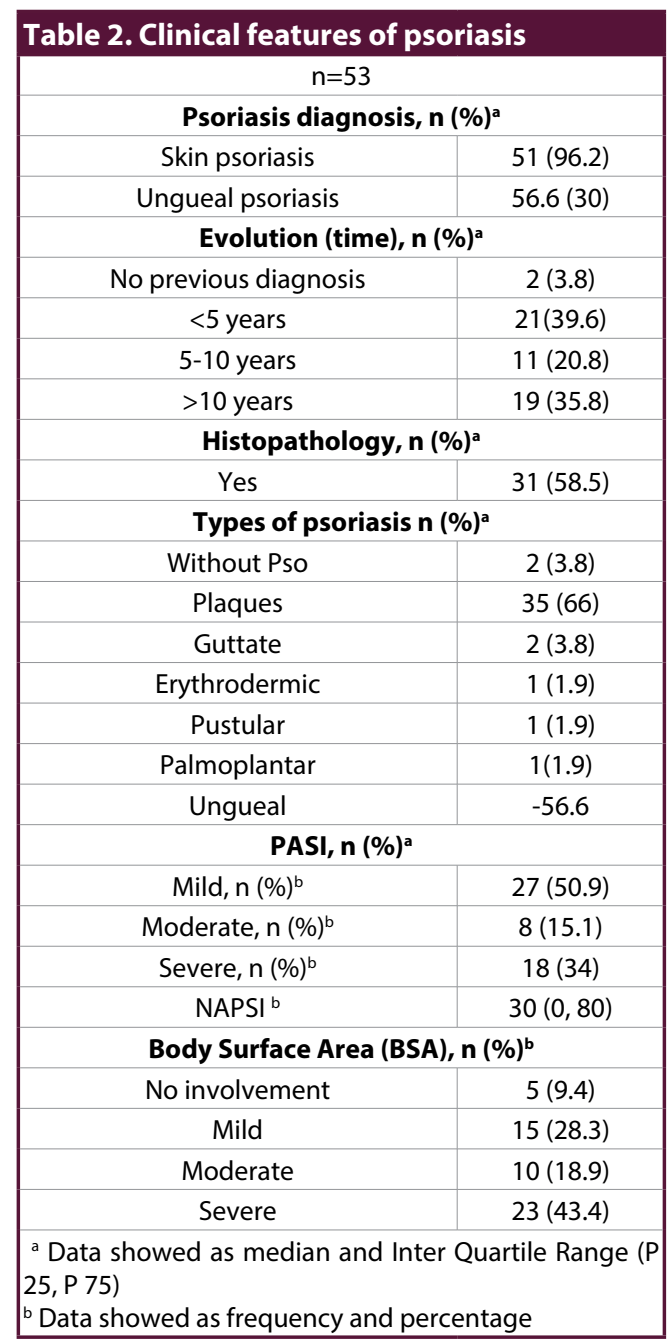

presented nail psoriasis. The median PASI score was $5.4(1.5,16.4) ; 61.3 \%$ of the participants had a mild disease, 6.5\% [2] had moderate disease and $32.3 \%[8]$ had severe disease. The median of the NAPSI score was 45 . The axial PsA was positive in $51.6 \%$ subjects. Peripheral disease was present in $71 \%$ of the participants. To determine the severity of psoriatic arthritis according to the DAS 28 classification, we found that 3.2\% [1] presented low activity, 22.6\% [7] showed moderate activity, and $45.2 \%$ presented severe activity. The total of subjects $100 \%$ with peripheral disease did not meet criteria for MDA (Minimal Disease Activity). Regarding the score of HAQ instrument to assess quality of life, found a median of $1.38(1.10,2.25) .100 \%$ of patients with psoriatic arthritis met CASPAR criteria. In Table 5 it is possible to observe the risk factors for the development of severe psoriatic arthritis. In the logistic regression model without adjustment, we found an OR of 1.45 for the metabolic syndrome variable (95\% CI 0.47 , 4.52), $\mathrm{p}=0.518$. For the variable of dyslipidemia, there was an OR of 0.93 (95\% CI $0.31,2.79)$, $\mathrm{p}=0.900$. The variable enthesitis showed an OR of 31 (95\% CI 5.73, 168), $\mathrm{p}=0.001$ and the variable obesity showed an OR of 1.35 (95\% CI $0.44,4.08), p=0.569$. In the adjusted regression model, the only variable representing a risk factor associated with the severity of psoriatic arthritis was the presence of enthesitis, with an OR of 32.2 (95\% CI 5.6, 183.7), $\mathrm{p}=0.001$. The other variables like metabolic syndrome, dyslipidemia and obesity were not predictive of severity in patients with psoriatic arthritis.

\section{Discussion}

The psoriasis and psoriatic arthritis clinic as a unique multidisciplinary evaluation model of 5 expert specialists in the same patient visit and in the same center, at the same time, makes it unique as a management strategy in psoriasis. The aim of this review is to demonstrate that there is a strong recommendation for multidisciplinary management, however this type of models currently only includes the dermatologist and rheumatologist as a part of the multidisciplinary approach. Given the complexity of this disease that includes comorbidities beyond the skin and joints like obesity and psychiatric illness among others, a medical team that can provide a more comprehensive intervention in this type of patients could be of great benefit. In our clinic each specialist is guided by a strict procedure manual that objectively measures the severity of the disease based on validated scales to implement management strategies from all areas of evaluation. The current description of these 53 patients is only the clinical characterization of the first visit in our clinic, however our objectives go beyond clinical features of patients with psoriasis.

In our patients we found a prevalence rate of metabolic syndrome of $35.8 \%$, according to Paschoal RS et al. [9], in a cross sectional study performed in 90 patients, they found a prevalence rate of metabolic syndrome of $43.3 \%$, a value very similar to our results. Is important to mention that our center has two years since it was opened so we expected to admit more patients and it is expected that the rates that we showed could change. This was the first study in Mexico that evaluated comorbidities, this is very important because there are other authors that have showed that comorbidities in psoriatic arthritis patients are an important burden in the health system $[8,10]$. Shah K et al. [10], showed a prevalence rate of $35 \%$ of dyslipidemia, 


\begin{tabular}{|c|c|c|c|}
\hline & $\begin{array}{l}\text { Psoriatic Arthritis } \\
\text { diagnosis } \\
\mathbf{n}=31\end{array}$ & $\begin{array}{l}\text { Without arthritis } \\
\qquad n=22\end{array}$ & $\mathbf{p}$ \\
\hline Age (years) ${ }^{a}$ & $42(35,58)$ & $39.5(25,55.25)$ & 0.184 \\
\hline $\begin{array}{l}\text { Sex, } \mathbf{n}(\%)^{c} \\
\text { Female }\end{array}$ & $22(71)$ & $11(50)$ & 0.121 \\
\hline Psoriasis in relatives, $\mathbf{n}(\%)^{c}$ & $8(25.8)$ & $6(27.6)$ & 0.905 \\
\hline Spondyloarthropathy in relatives, $\mathbf{n}(\%)^{c}$ & $6(19.4)$ & 0 & 0.035 \\
\hline \multicolumn{4}{|c|}{ Tobacco n (\%)c } \\
\hline Yes & $8(25.8)$ & $6(27)$ & \\
\hline No & $22(71)$ & $13(59.1)$ & 0.564 \\
\hline $\begin{array}{c}\text { Alcohol consumption, } \mathbf{n}(\%) \\
\text { Yes }\end{array}$ & $7(22.6)$ & $10(45.5)$ & 0.079 \\
\hline $\begin{array}{l}\text { Exercising, } \mathbf{n}(\%)^{\mathbf{c}} \\
\text { Yes }\end{array}$ & $8(25.8)$ & $7(31.8)$ & 0.632 \\
\hline $\begin{array}{c}\text { Healthy eating habits, } \mathbf{n}(\%)^{c} \\
\text { Yes }\end{array}$ & $7(22.6)$ & $5(22.7)$ & 0.99 \\
\hline \multicolumn{4}{|c|}{ Nutritional state (\%)c } \\
\hline Normal & $9(29)$ & $9(40.9)$ & \\
\hline Overweight I & $4(12.9)$ & $2(9.1)$ & \\
\hline Overweight II & $9(29)$ & $3(13.6)$ & 0.371 \\
\hline Grade I Obesity & $6(19.4)$ & $5(22.7)$ & \\
\hline Grade II Obesity & $1(3.2)$ & $3(13.6)$ & \\
\hline Grade III Obesity & $2(6.5)$ & 0 & \\
\hline \multicolumn{4}{|c|}{ Comorbidities, $\mathbf{n}(\%)^{c}$} \\
\hline Diabetes & $6(19.4)$ & $2(9.1)$ & 0.445 \\
\hline Hypertension & $7(22.6)$ & $19(86.4)$ & 0.412 \\
\hline Cardiovascular disease & $3(9.7)$ & $2(9.1)$ & 0.099 \\
\hline Metabolyc Syndrome & $12(38.7)$ & $7(31.7)$ & 0.606 \\
\hline Dyslipidemia & $16(51.6)$ & $11(50)$ & 0.202 \\
\hline \multicolumn{4}{|c|}{ Goldberg's depression test $\mathbf{n}(\%)^{c}$} \\
\hline Depression & $11(35.5)$ & $5(22.7)$ & 0.319 \\
\hline \multicolumn{4}{|c|}{ Goldberg's anxiety test $n(\%)^{c}$} \\
\hline Anxiety & $18(58.1)$ & $8(36.4)$ & 0.119 \\
\hline Cholesterol mg/dL ${ }^{b}$ & $188+40.10$ & $181.51+30.38$ & 0.517 \\
\hline $\mathrm{HDL} \mathrm{mg/dL^{b }}$ & $41.91+11.64$ & $45.27+9.3$ & 0.269 \\
\hline Glucose $\mathrm{mg} / \mathrm{dL}^{\mathrm{a}}$ & $97(87,104)$ & $92(72.2,106.2)$ & 0.481 \\
\hline $\mathrm{LDL}^{\mathrm{a}}$ & $110(90,125)$ & $120(90.7,126.5)$ & 0.935 \\
\hline Triglycerides & $145(115,189)$ & $144.5(111,214.7)$ & 0.821 \\
\hline $\mathrm{ESR}^{\mathrm{a}}$ & $27(14,38)$ & $12(10,16.2)$ & 0.001 \\
\hline $\mathrm{ESR}>15^{\mathrm{c}}$ & $23(74.2)$ & $6(27.3)$ & 0.001 \\
\hline Rheumatoid Factor $+\mathrm{c}$ & $3(9.7)$ & $1(4.5)$ & 0.633 \\
\hline \multicolumn{4}{|c|}{$\begin{array}{l}\text { a Data showed as median and intequartile range (P25, P75), U Mann Whitney } \\
\text { b Data showed as median and standard deviation, t Student } \\
\text { c Data showed as frequency and percentage, } \mathrm{X}^{2}\end{array}$} \\
\hline
\end{tabular}

$31 \%$ of hypertension, $15 \%$ of depression and $13 \%$ of diabetes mellitus vs. 22, 31, 15 and $13 \%$ respectively. In our center we measured anxiety, with a prevalence rate of $49.1 \%$. The prevalence rate of obesity was $16.4 \%$ vs. $32.1 \%$, this makes sense given the high prevalence of obesity that exists in our Country. This is very important because obesity is comorbidity that difficult the management of the disease and the response to the treatment. The specific scales of each specialist and the results in the following visits will show if this multidisciplinary model can really change the course, quality of life and management in patients with psoriasis. This management has been recommended previously by the Canadian Dermatology-Rheumatology Comorbidity Initiative [11].

This is the first overview of our clinic to better understand the problem of psoriasis, however the model is designed for the management not only of psoriasis and psoriatic arthritis, but also 
associated comorbidities looking for a real impact on the management and quality of life of our patients with psoriasis, thus we strongly believe that our multidisciplinary model evaluated over time, will make a difference in the management of this complex disease. Our multidisciplinary model has identified psoriasis as a disease

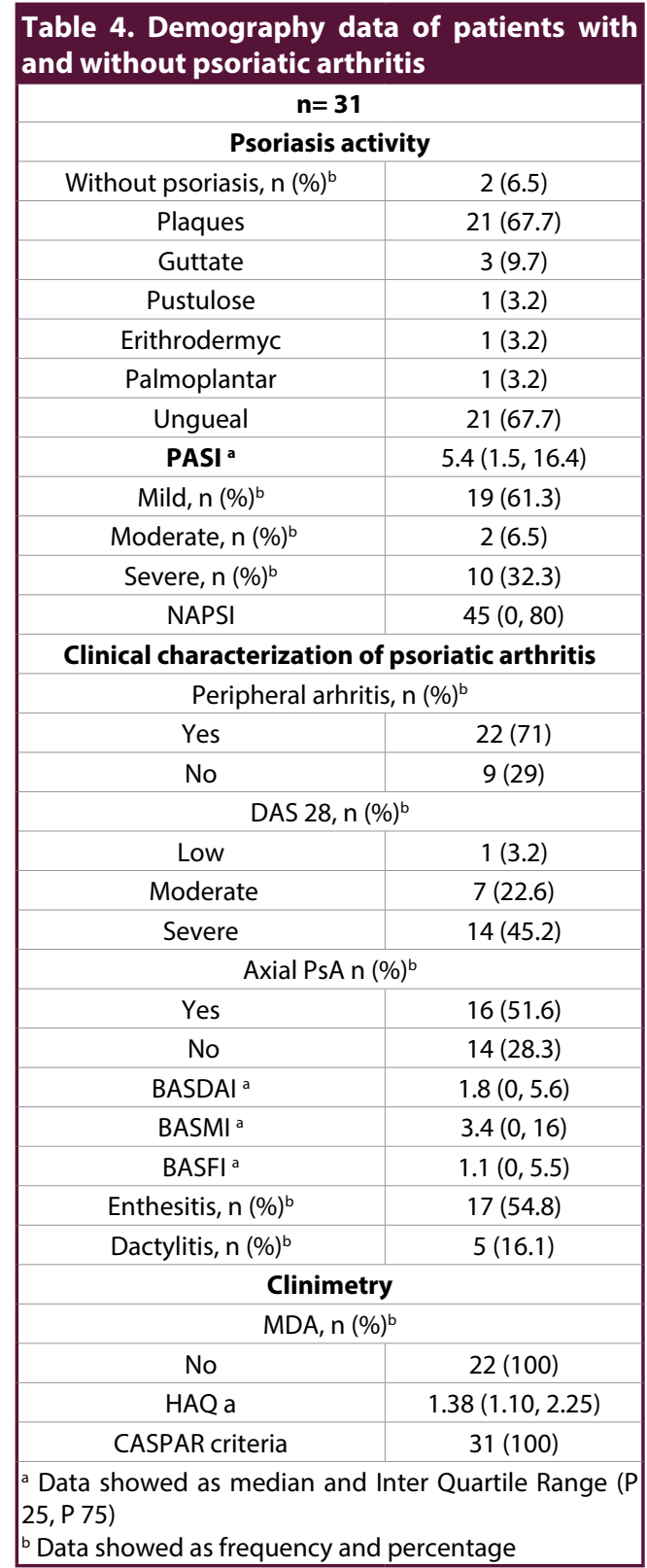

with an unpredictable course, which requires evaluation by a multidisciplinary model, not limited to the dermatologist and rheumatologist only. This has been mentioned previously in the recommendations by the Spanish group [12]. The first recommendation indicates the importance of the management of the psoriatic arthritis by both dermatologist and rheumatologist. Our center is the first that include a nutritionist, internal medicine physician and a psychiatrist. All of them evaluated to the 53 patients since their first visit.

\section{Conclusions}

In our series of 53 patients, we found that nearly half of the patients presented a severe form of psoriasis with significant quality of life impairment, evaluated by three dermatologists; the percentage of patients with psoriatic arthritis was higher than expected. Since $58 \%$ of the patients evaluated met CASPAR criteria, with both axial and peripheral manifestations, interpretation and classification was determined by rheumatologist. As already described in several studies, we have found a high percentage of comorbidities such as metabolic syndrome, diabetes mellitus, hypertension, dyslipidemia and obesity, assessed by internal medicine physician and nutrition expert. We also found a high frequency of psychiatric illness, anxiety being the most frequent manifestation in this series of patients. Psychiatric evaluations were performed using validated scales. All of the clinical assessments were performed under the same protocols in the same place and visit by the 5 specialties, at the time in the same patient visit. The main limitation of our study is the small sample that fails to demonstrate if the presences of comorbidities have an impact in the severity of skin and joint disease. However, this study is the beginning of the follow-up of these patients, we will evaluate the results to long term in order to show the response to the different treatments, multidisciplinary management and its impact in the prevalence and incidence rates of comorbidities. This clinic is a practical private place so, it is important to mention that the cost of the management of the patients is very low

\begin{tabular}{|c|c|c|c|c|c|c|}
\hline Variables & $\mathbf{O R}^{\mathrm{a}}$ & $\mathrm{Cl} 95 \%$ & $\mathbf{p}$ & $O R^{b}$ & $\mathrm{Cl95 \%}$ & $\mathbf{p}$ \\
\hline Metabolic syndrome & 1.45 & $(0.47,4.52)$ & 0.518 & 1.08 & $(0.13,8.96)$ & 0.939 \\
\hline Dislypidemia & 0.93 & $(0.31,2.79)$ & 0.9 & 0.61 & $(0.07,4.80)$ & 0.64 \\
\hline Enthesitis & 31 & $(5.73,168)$ & 0.001 & 32.2 & $(5.6,183.7)$ & 0.001 \\
\hline Obesity & 1.35 & $(0.44,4.08)$ & 0.569 & 1.76 & $(0.30,10.2)$ & 0.528 \\
\hline
\end{tabular}


because the purpose of the clinic is performing clinical research in the occident of the country. It is important to mention that this is the first type of study in Mexico.

Acknowledgments

Dr. Lilian Torres Made, Internal Medicine physician

Dr. Janeth Aguilar Liaison, Psychiatrist

Lic. Rossdali Sanchez, Clinical nutritionist

Dr. Gabriela Garcia, Rheumatologist Ultrasonographer

Multidisciplinary model for psoriasis and psoriatic arthritis clinic team

Dr. Roxana Rodríguez by the methodological advice

Special acknowledgments to Dr. Helena Marzo Ortega, from the University of Leeds, UK, who has supported and followed this project since its beginning.

Funding

Dr. Ivonne Roy García MsC Clinical Research Training Center Division of Research Development. Statistical analysis of the project of the multidisciplinary model for psoriasis and psoriatic arthritis clinic team.

\section{Competing and conflicting Interests}

The authors declare that they have no competing interest.

\section{References}

1. Nestle FO, Kaplan DH, Barker J. Psoriasis. N. Engl. J. Med. 361(5), 496-509 (2009).

2. Chang CA, Gottlieb AB, Lizzul PF. Management of psoriatic arthritis from the view of the dermatologist. Nat. Rev. Rheumatol. 7(10), 588-98 (2011).

3. Gladman DD, Antoni C, Mease $\mathrm{P}$ et al. Psoriatic arthritis: epidemiology, clinical features, course, and outcome. Ann. Rheum. Dis. 64Suppl(2), 14-7 (2005).
4. Eder $\mathrm{L}$, Chandran $\mathrm{V}$, Shen $\mathrm{H}$ et al. Incidence of arthritis in a prospective cohort of psoriasis patients. Arthritis. Care. Res. (Hoboken). 63(4), 619-22 (2011).

5. Mease PJ, Armstrong AW. Managing patients with psoriatic disease: the diagnosis and pharmacologic treatment of psoriatic arthritis in patients with psoriasis. Drugs. 74, 423-441 (2014).

6. Kane D, Stafford L, Bresnihan B et al. A prospective, clinical and radiological study of early psoriatic arthritis: an early synovitis clinic experience. Rheumatology. (Oxford). 42, 1460-1468 (2003).

7. Takeshita J, Grewal S, Langan SM et al. Psoriasis and comorbid diseases: Implications for management. J. Am. Acad. Dermatol. 76(3), 393-403 (2017).

8. Queiro R, Coto P, Joven B et al. Current Status of Multidisciplinary Care in Psoriatic Arthritis in Spain: NEXUS 2.0 Project. Reumatol. Clin. S1699258X(18)30030-5 (2018).

9. Paschoal RS, Silva DA, Cardili RN et al. Metabolic syndrome, C-reactive protein and cardiovascular risk in psoriasis patients: a cross-sectional study. An. Bras. Dermatol. 93(2), 222-228 (2018).

10. Shah K, Paris M, Mellars L et al. Real-world burden of comorbidities in US patients with psoriatic arthritis. RMD Open. 3(2), e000588 (2017).

11. Roubille C, Richer V, Starnino $\mathrm{T}$ et al. Evidencebased Recommendations for the Management of Comorbidities in Rheumatoid Arthritis, Psoriasis, and Psoriatic Arthritis: Expert Opinion of the Canadian Dermatology-Rheumatology Comorbidity Initiative. $J$. Rheumatol. 42(10), 1767-80 (2015).

12. Torre-Alonso JC, Carmona L, Moreno $\mathrm{M}$ et al. Identification and management of comorbidity in psoriatic arthritis: evidence- and expert-based recommendations from a multidisciplinary panel from Spain. Rheumatol. Int. 37(8), 1239-1248 (2017). 